Rev. Biol. Trop. 46(4): 1159-1161, 1998

www.ucr.ac.cr www.ots.ac.cr www.ots.duke.edu

\title{
Reproducción asincrónica de Sceloporus palaciosi (Sauria: Phrynosomatidae) en México, con comentarios sobre sus ventajas y regulación
}

\author{
Fausto R. Méndez' y Maricela Villagrán²
}

Instituto de Biología y ${ }^{2}$ Facultad de Ciencias, Universidad Nacional Autónoma de México, C.P. 04510, A.P. 70-515, México 20 Distrito Federal, México.

Recibido 13-II-1998. Corregido 2-VII-1998. Aceptado 29-VII-1998.

\begin{abstract}
Fall reproduction is a common pattern in viviparous lizards from Mexican mountains. The reproductive pattern of Sceloporus palaciosi is seasonal but asynchronous between sexes. Males show testicular development in May and June whereas females ovulate in October. We suggest that the same environmental stimulous to reproduction occur twice a year, but the internal system determines the response of both sexes to different seasons, and fall stimulation allows early sexual maturity in females.
\end{abstract}

Key words: Reproductive cycle, viviparity, sexual maturity, Sceloporus palaciosi.

Dentro del género Sceloporus, el grupo grammicus presenta una amplia distribución y todos sus miembros son vivíparos (Méndez et $a l$. en prensa). En este grupo se presentan ambos patrones reproductores: sincrónicos y asincrónicos (Guillette y Méndez 1993). Sceloporus palaciosi es una especie del grupo grammicus que se distribuye en el centro de México, del cual se desconoce su biología. Este trabajo describe su ciclo reproductor, plantea a que obedece la fenología reproductora y cuales son sus ventajas.

La población estudiada se localiza en la Sierra del Ajusco, al sur de la Ciudad de México, entre 3200 y $3400 \mathrm{msnm}$. Se colectaron mensualmente de 10 a 15 especímenes de cada sexo de $S$. palaciosi de enero a diciembre de
1984, se les determinó la longitud hocico cloaca (LHC) y la condición reproductora. En los machos fué obtenido con base en el volumen testicular (calculado con la fórmula de un elipsoide: $\mathrm{VT}=4 / 3 \mathrm{II}\left(\mathrm{a}^{\mathrm{b}} \mathrm{b}\right)$, en donde: $\mathrm{VT}=$ volumen testicular, $\mathrm{a}=1 / 2$ diámetro testicular menor y $b=1 / 2$ del diámetro testicular mayor $y$ en las hembras se obtuvo midiendo el diámetro del folículo ovárico mayor, definiendo previtelogénesis, vitelogénesis o preñez. Se determinaron cambios significativos mensuales a través del año para el volumen testicular y el diámetro folicular mediante un Análisis de Covarianza (ANCOVA) y una prueba de intervalos múltiples (Bruning y Kintz 1977).

Se elaboró el climograma (Fig. 1A) utilizando los datos de temperatura media y 


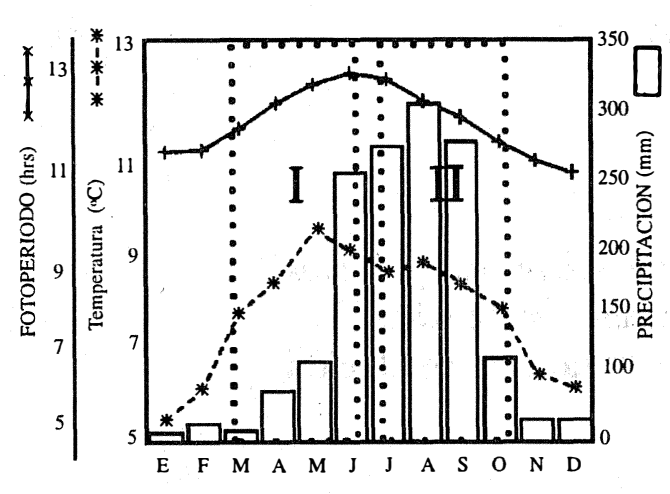

A.

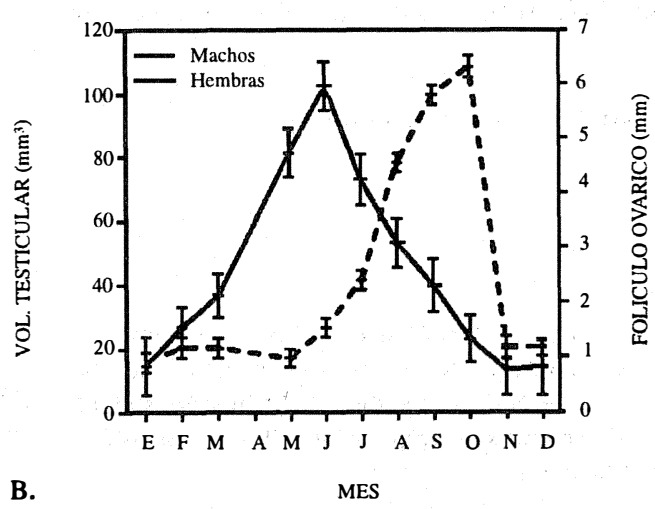

B.

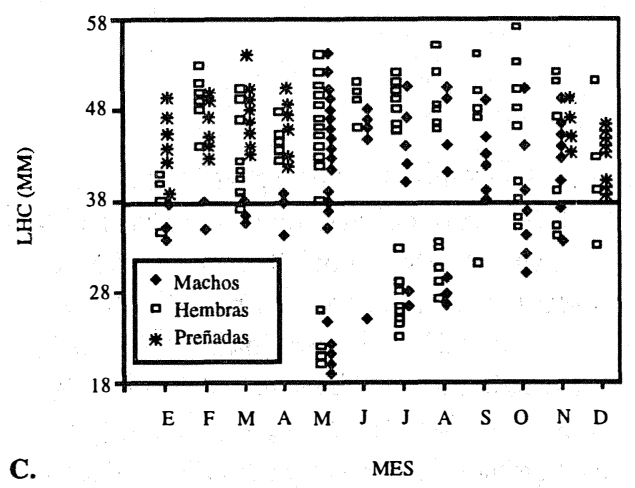

Fig. 1. A. Climograma de Monte Alegre, Ajusco. I y II sugieren las temporadas de estimulación ambiental para la reproducción. B. Ciclo reproductor de machos y hembras de Sceloporus palaciosi. C. Variación de las tallas de $S$. palaciosi a lo largo del año. La línea indica la talla a la cual las hembras alcanzan la madurez sexual. precipitación promedio de cada mes de la estación meteorológica de Monte Alegre, Ajusco y la cantidad de horas luz promedio mensual para la Ciudad de México (Anónimo 1986). El coeficiente de determinación entre los valores gonadales (volumen testicular y diámetro folicular) y los datos ambientales mensuales (precipitación, temperatura y fotoperíodo) se utilizó para determinar la asociación entre la reactivación gonadal y los factores climáticos, aplicándose posteriormente una prueba de Fisher (Bruning y Kintz 1977). Para evaluar el crecimiento y la madurez sexual, se graficó la longitud hocico cloaca (LHC) de los especímenes colectados a lo largo del año.

$S$. palaciosi presenta un ciclo reproductor asincrónico (Fig. 1B). El volumen testicular presentó cambios significativos durante el año $(\mathrm{F}=(11,95)=12.79, \mathrm{p}<0.0001)$. De noviembre a enero muestra bajos volúmenes (inactividad), mientras que el incremento se inicia en febrero (reactivación). La máxima actividad reproductora ocurre durante mayo y junio y la regresión gonadal de julio a octubre, indicando un patrón de actividad primavera-verano. El incremento testicular se correlacionó positivamente con la temperatura $\left(\mathrm{r}^{2}=58.0, \mathrm{p}<0.0001\right)$, con la precipitación $\left(\mathrm{r}^{2}=57.9, \mathrm{p}<0.0001\right)$ y con el fotoperiodo $\left(\mathrm{r}^{2}=61.6, \mathrm{p}<0.0001\right)$.

En las hembras, el diámetro del folículo ovárico mayor mostró cambios significativos a lo largo del año $(F[11,91]=122.84, p<0.0001)$. La fase de inactividad ocurre de noviembre a mayo, coincidiendo con la gestación. Las hembras previtelogénicas se encuentran en mayo y junio y la etapa vitelogénica sucede de junio a octubre. En noviembre ocurre la ovulación y de diciembre a abril se encuentran preñadas, indicando que la máxima actividad gonadal ocurre en el otoño (Fig. 1B). Durante la vitelogénesis, el diámetro folicular se correlacionó negativamente con la temperatura $\left(r^{2}=70.6, p<0.0001\right)$ $y$ con el fotoperiodo $\left(r^{2}=75.2, p<0.0001\right)$.

La distribución de tamaños en machos y hembras indica que existe un rápido crecimiento en los primeros meses de vida (Fig. 1C), alcanzando las hembras $38 \mathrm{~mm}$ de LHC a los 7 meses de edad e iniciando la preñez. 
El ciclo reproductor asincrónico de $S$. palaciosi es similar al que presentan otras poblaciones de montaña de Sceloporus grammicus y al de otras especies de Sceloporus vivíparas (Guillette y Méndez 1993), lo que parece ser una estrategia favorecida en zonas templadas. Una ventaja de este ciclo es que las crías nacen en mayo, cuando inicia la temporada de lluvias (Fig. 1A), y esta época coincide con una alta disponibilidad de alimento (Méndez et al. 1995).

Guillette y Casas-Andreu (1980) proponen que la actividad reproductora en ambos sexos de $S$. grammicus responde a diferentes estímulos ambientales. Jones (1981) menciona que la luz estimuladora de la reproducción se presenta dos veces al año, y proponemos que podría ser extensivo para la variación de la temperatura a lo largo del año. Con base en lo anterior sugerimos que hay dos fases de estímulo: una entre primavera y verano (Fig. 1A, I) y otra entre verano y otoño (Fig. 1A, II). Los machos de $S$. palaciosi parecen responder a la primera fase, al igual que la mayoría de especies ovíparas y vivíparas (Méndez et al. en prensa), y las hembras no responden a ésta fase por estar preñadas. La segunda fase parece estimular la vitelogénesis en las hembras, cuando los factores climáticos presentan valores similares a los de marzo-junio, pero con tendencia decreciente. Esta segunda estimulación parece ser clave en el alcance de la madurez sexual de las hembras de $S$. palaciosi.

Dunham et al. (1988) mencionan que la maduréz sexual de los lacertilios se alcanza desde los 5.5 hasta los 30 meses y consideran que las especies ovíparas maduran más tempranamente que las vivíparas (14.9 vs 17.9 meses respectivamente). Sin embargo, en $S$. palaciosi la segunda fase de estimulación es vital en la estrategia reproductora, ya que las hembras que alcanzan tallas mayores de $38 \mathrm{~mm}$ a los 7 meses de vida se encuentran preñadas (Fig. 1C). La madurez sexual temprana se ha registrado previamente en otras especies vivíparas del género (Méndez et al. en prensa). El aprovechamiento de la época de mayor cantidad de alimento (promovido por las lluvias) y de una segunda fase de estimulación permite crecer e iniciar la reproducción tempranamente. De esta manera, algunas hembras, pueden reproducirse antes de alcanzar un año de edad. Lo anterior sugiere que en las especies de este género de ambientes templados se combinan, dentro la misma estrategia reproductora, la viviparidad y la madruez sexual temprana.

\section{AGRADECIMIENTOS}

A DGAPA, UNAM por el apoyo para la estancia en Virginia Polytechnic Institute \& State Univestity y a CONACYT de México proyectos $2155 \mathrm{~N} 9302$ y 4171 N9405.

\section{REFERENCIAS}

Anónimo. 1986. Anuario del Observatorio Meteorológico Nacional. Instituto de Astronomía, Universidad Nacional Autónoma de México, México, D.F.

Bruning J.L. \& B. Kintz 1977. Computational handbook of statistics. Scott Foresman, Glenview, Illinois. 308 p.

Dunham, A.E., D.B. Miles \& D.N. Reznick. 1988. Life history patterns of squamate reptiles. p. 441-522. In C. Gans \& R.B. Huey (eds.). Biology of the Reptilia. Vol. 16, Ecology B. Defense and Life History. Wiley, Nueva York.

Guillette, L.J., Jr. \& G. Casas-Andreu. 1980. Fall reproductive activity in the high altitude Mexican lizard Sceloporus grammicus microlepidotus. J. Herpetol. 14:143-147.

Guillette, L.J., Jr. \& F.R. Méndez. 1993. The reproductive cycle of the viviparous Mexican lizard Sceloporus torquatus. J. Herpetol. 27:168-174.

Jones, R.E. 1981. Mechanisms controlling seasonal ovarian quiescence. p. 205-234. In Schwartz N.B. \& M. Hunzicker-Dunn (eds.). Dynamics of Ovarian Function. Raven, Nueva York.

Méndez, F.R , R. Sánchez-Trejo \& O. Cuellar. 1995. Reproductive differences betwen sympatric oviparous and viviparous Mexican spiny lizards. Biogeographica 71:61-67.

Méndez, F.R., M. Villagrán \& R. M. Andrews. 1998. Evolution of viviparity in the lizard genus Sceloporus. Herpetologica (en prensa). 